\title{
Understanding urologic scientific publication patterns and general public interests on stone disease: lessons learned from big data platforms
}

\author{
Giovanni S. Marchini ${ }^{1,2} \cdot$ Kauy V. M. Faria $^{1} \cdot$ Felippe L. Neto $^{1} \cdot$ Fábio César Miranda Torricelli $^{1,2} \cdot$ Alexandre Danilovic $^{1}$. \\ Fábio Carvalho Vicentini ${ }^{1}$. Carlos A. Batagello ${ }^{1,2}$. Miguel Srougi ${ }^{1}$. William C. Nahas ${ }^{1}$ - Eduardo Mazzucchi ${ }^{1}$
}

Received: 10 June 2020 / Accepted: 1 October 2020 / Published online: 27 October 2020

(c) Springer-Verlag GmbH Germany, part of Springer Nature 2020

\begin{abstract}
Purpose To analyse patterns of stone disease online information-seeking behaviours in the United States and to correlate with urological literature publication aspects.

Methods To compare Relative Search Volume (RSV) among different twelve preselected urologic keywords we chose "United States" as country and "01/01/2009-31/12/2018" as time range on Google Trends (GT). We defined "ureteroscopy" as a reference and compared RSV against it for each term. RSV was adjusted and normalized in a scale 0-100. Trend presence was evaluated by Mann-Kendall Test and magnitude by Sen's Slope Estimator (SS). Weather influence on RSV was also investigated by comparison of the ten hottest versus ten coldest states. Pearson correlation analysis was performed between number of Pubmed publications and RSV for each term over time.

Results We found an upward tendency $(p<0.01)$ for most terms. Higher temporal trends were seen for "kidney stone" ( $\mathrm{SS}=0.36)$, "kidney pain" ( $\mathrm{SS}=0.39)$ and "tamsulosin" $(\mathrm{SS}=0.21)$. Technical treatment terms had little search volumes and no increasing trend. States with hotter weather showed higher mean RSV for "kidney stone" than colder ones. There was little correlation between GT and Pubmed for most terms, with the exception of "kidney stone" $(R=0.89 ; p<0.01)$, "URS" $(R=0.81 ; p<0.01)$, and "laser lithotripsy" $(R=0.74 ; p=0.01)$.

Conclusion There was a significant increase in online search for medical information related to stone disease. Citizens tend to look for generic terms related to symptoms or the disease itself. States with hotter weather show higher RSV than colder states. There is a discrepancy between public and medical community medical terms.
\end{abstract}

Keywords Google Trends $\cdot$ Lithiasis $\cdot$ Health $\cdot$ Public Interest $\cdot$ Epidemiology $\cdot$ Pubmed

$\begin{array}{ll}\text { Abbreviations } \\ \text { GT } & \text { Google Trends } \\ \text { PCNL } & \text { Percutaneous Nephrolithotomy } \\ \text { RSV } & \text { Relative Search Volume }\end{array}$

Electronic supplementary material The online version of this article (https://doi.org/10.1007/s00345-020-03477-5) contains supplementary material, which is available to authorized users.

Giovanni S. Marchini marchinism@gmail.com

1 Section of Endourology, Division of Urology, Hospital das Clinicas, University of Sao Paulo Medical School, Av. Dr. Enéas Carvalho de Aguiar 255, Cerqueira César, 05403-900 Sao Paulo, Brazil

2 Glickman Urological and Kidney Institute, Cleveland Clinic, Cleveland, OH, USA
SS Sen's Slope Estimator

SWL Extracorporeal Shockwave Lithotripsy

URS Ureteroscopy

US United States

\section{Introduction}

Urinary lithiasis is a common disease with an approximated prevalence of $7-12 \%$ around the world $[1,2]$. Over the past 15 years, the prevalence of urinary stone disease has nearly doubled in United States (US) [3]. Medical expulsive therapy, ureteroscopy (URS), percutaneous nephrolithotomy (PCNL) and extracorporeal shockwave lithotripsy (SWL) are the most common treatment modalities for kidney and ureteral stones $[4,5]$. A shift from open to less-invasive 
techniques was seen in the last decades and as a consequence, the dedicated medical literature increased [6].

As stone disease burden ascents, online internet search for information regarding the theme upsurges globally. The United States (US) is the fourth largest country in the world by land area with over 312 million internet users as of 2018, being one of the largest online markets worldwide. In 2019, just ten percent of US adults reported that they did not use the internet [7]. Just recently, online strategies and internet trend search gathering tools have been developed to provide useful information for monitoring global and regional health seeking behaviour patterns, epidemiology, aetiology and treatment of various medical conditions, including urolithiasis [8]. Google Trends (GT) is one of the most important of these tools, in which internet queries are catalogued and this indexed information is available to the public. The big data database provided by GT can reveal patterns in health information-seeking behaviour on a population level, allowing development of target information to the public $[8,9]$.

The main purpose of this study was to analyse patterns of stone disease information-seeking behaviours in the US to understand how patients look for reliable information in this matter. Second, we aimed to study the influence of weather on online search patterns to trace a parallel with stone disease incidence. Finally, we aimed to compare the GT findings to the urological literature publication aspects.

\section{Methods}

\section{Data Interpretation}

Google Trends is a web service offered by Google Inc. that keeps track of online keywords interest according to country or region over a selected time period. In addition, the search of distinguish terms in different regions can be compared simultaneously. Data are downloaded from the web in ".csv" format and adjusted as follows: search results are proportionate to the time and location of a query; each data point is divided by the total absolute searches of the geography and time range it represents, to compare relative popularity. Otherwise places with the most search volume would always be ranked highest. The resulting numbers are then scaled on a range of $0-100$ based on a topic's proportion to all searches on all topics.

Google's normalized trends data are the most useful parameter for storytelling, i.e., when we look at a topic search interest over time, we are looking at that interest as a proportion of all searches on all topics on Google at that time and location [10]. Therefore, GT provides a Relative Search Volume (RSV) which is a sampled estimate of a particular query share according to location and time normalized by the highest query of the period in a $1-100$ scale. Up to five multiple terms analysis is allowed for query comparison.

\section{Data collection and processing}

\section{Google trends}

To compare RSV among different urologic keywords, we have used "United States" as country, "01/01/2009-31/12/2018" as time-range, "All Categories" as category and "Web Search" as type of search.

English terms were first selected after a recent study which explored their popularity [8]. A more comprehensive assessment of expressions was included and the final keywords for each term were chosen by an expert based on multiple attempts until one was found to capture the greatest RSV for the period.

To compare more than GT's limit of five words in the US, we defined "lithotripsy" as a reference and downloaded RSV comparisons against it for each term. We then adjusted the RSV numbers by the reference and normalized them by the highest RSV for the period in a scale $0-100$.

The final selection included the following twelve keywords: "kidney stone";"renal stone";"kidney stone surgery”;"renal colic";"kidney pain”;"“ureteroscopy”;“extr acorporeal shockwave lithotripsy";"percutaneous nephroli thotomy”;"tamsulosin";"kidney stent","lithotripsy”;"laser lithotripsy".

To compare trends among different US states from 2009 to 2018, we have downloaded yearly RSV by "sub-region" for the term with the highest RSV on the previous analysis, i.e. "kidney stone". For comparison, the states were further divided in two groups according to temperature and weather estimates:

- Ten hottest states: Florida, Hawaii, Louisiana, Texas, Georgia, Mississippi, Alabama, South Carolina, Arkansas, Arizona;

- Ten coldest states: Alaska, North Dakota, Maine, Minnesota, Wyoming, Montana, Vermont, Wisconsin, New Hampshire, Michigan.

\section{Pubmed}

The number of studies published on the online medical search database was downloaded from Pubmed service using the advanced tool with the same twelve terms selected for Google Trends in the 'Title' filter. We have included publications in the same 2009-2018 range. The absolute number of publications per year was considered in the analysis. 


\section{Statistical analysis}

Trend presence was evaluated using the Mann-Kendall test and magnitude was estimated using the Sen's Slope Estimator (SS). Both of them apply to non-parametric data and were used to evaluate trends for Pubmed and GT over time.

Correlation analysis was done using Pearson method, which is the standard method used by Google in Correlate Service, to address correlations between Pubmed and GT data.

GT comparison between groups of states was done with $T$ Test (normal distribution). Continuous data were detailed as mean and standard deviation. Statistical analysis was done in $R$ version 3.5.1. Significance was set at $p<0.05$.

\section{Results}

\section{Temporal Trends on Google}

Year trend analysis revealed an upward trend $(p<0.01)$ in most of the researched terms as described in Table $1 \mathrm{a}$. Figure 1a depicts the data in regard to Google Trend RSV in the US. Higher temporal trends were seen for "kidney Stone", "kidney pain" and "tamsulosin". "Tamsulosin" had an expressive search volume in $2018(\mathrm{RSV}=30.70)$ compared to other terms with a tenfold increase over the tenyear period analysis (2009 RSV =3.07). Interestingly, "renal colic" and "renal stone" had low search volumes.

Treatment terms which demanded more knowledge of medical vocabulary as "SWL", "laser lithotripsy", and "PCNL" had little search volumes and no increasing trend, remaining low in public interest during the ten-year analysis. The most looked-up terms related to surgical procedures were "lithotripsy" and "kidney stent". "Kidney stent" had a significant increase in search trend over time and had a relevant search volume in $2018(\mathrm{RSV}=4.97 ; \mathrm{S}=0.02 ; p<0.01)$.

\section{Pubmed publication patterns}

The number of publications varied considerably yearly for each included term. Figure $1 \mathrm{~b}$ depicts the volume of publications for each term per year. The expressions with the highest number of publications per year were "lithotripsy" (183.4), "PCNL" (176.9), and "URS" (79.3). On the contrary, the expressions with the lowest number of publications were "kidney pain" $(0.46)$ and "kidney stone surgery" $(0.8)$.

In regard to the temporal trend among publication patterns (Table 1b; Fig. 1b), there was a significant increase in studies with the respective words in the title: "PCNL", "URS", "lithotripsy", "kidney stone", "laser lithotripsy", "renal colic", and "renal stone". Other terms had no significant increase over time.

\section{Correlation analysis}

The volumes of publications on Pubmed and RSV on Google are demonstrated in Supplementary Fig. 1 in a $0-100$ scale to allow for visual comparison in regard to volume relevance. Not only the volume was different for most terms but also the temporal trend line. There was a significant strong positive correlation for the terms "kidney stone", "URS", and "laser lithotripsy". For all other terms, there was no significant correlation.

Specifically, for GT data, medical knowledge content as "Extracorporeal Shockwave Litotripsy", "Percutaneous Nephrolitothomy", "Litotripsy" had lower correlation with popular terms as "Kidney Pain" or "Kidney Stone". A more generic
Table 1 Temporal Trend measured by Mann-Kendall Sen's Slope Estimator (SS) for Google Trend enquiry (a) and Pubmed data (b); CCorrelation between databases

\begin{tabular}{|c|c|c|c|c|c|c|c|}
\hline \multicolumn{3}{|l|}{ A-Google Trends } & \multicolumn{3}{|l|}{ B-Pubmed } & \multicolumn{2}{|c|}{$\mathrm{C}$-Correlation } \\
\hline Query & SS & $p$ value & Query & SS & $p$-value & $(\mathrm{R})$ & $p$-value \\
\hline Kidney stone & 0.37 & $<0.01$ & Kidney stone & 4.71 & $<0.01$ & 0.89 & $<0.01$ \\
\hline Kidney pain & 0.40 & $<0.01$ & Kidney pain & 0.00 & 0.12 & 0.41 & 0.24 \\
\hline Renal stone & 0.00 & $<0.01$ & Renal stone & 1.50 & $<0.01$ & 0.43 & 0.21 \\
\hline Tamsulosin & 0.21 & $<0.01$ & Tamsulosin & 0.63 & 0.78 & 0.43 & 0.21 \\
\hline Renal Colic & 0.00 & 0.10 & Renal colic & 2.67 & 0.03 & 0.11 & 0.75 \\
\hline Kidney stone surgery & 0.02 & $<0.01$ & Kidney stone surgery & 0.16 & 0.06 & 0.43 & 0.21 \\
\hline PCNL & 0.00 & 0.27 & PCNL & 16.17 & $<0.01$ & 0.02 & 0.96 \\
\hline URS & 0.01 & $<0.01$ & URS & 8.85 & $<0.01$ & 0.81 & $<0.01$ \\
\hline SWL & 0.00 & 0.30 & SWL & 0.33 & 0.78 & 0.13 & 0.72 \\
\hline Laser lithotripsy & 0.00 & $<0.01$ & Laser lithotripsy & 3.00 & 0.01 & 0.74 & 0.01 \\
\hline Lithotripsy & 0.00 & $<0.01$ & Lithotripsy & 5.25 & 0.03 & 0.54 & 0.10 \\
\hline Kidney stent & 0.02 & $<0.01$ & Kidney stent & 1.00 & 0.47 & 0.42 & 0.22 \\
\hline
\end{tabular}

Values in bold indicate significance

$P C N L$ Percutaneous nephrolithotomy, URS Ureteroscopy, SWL Extracorporeal shockwave lithotripsy 


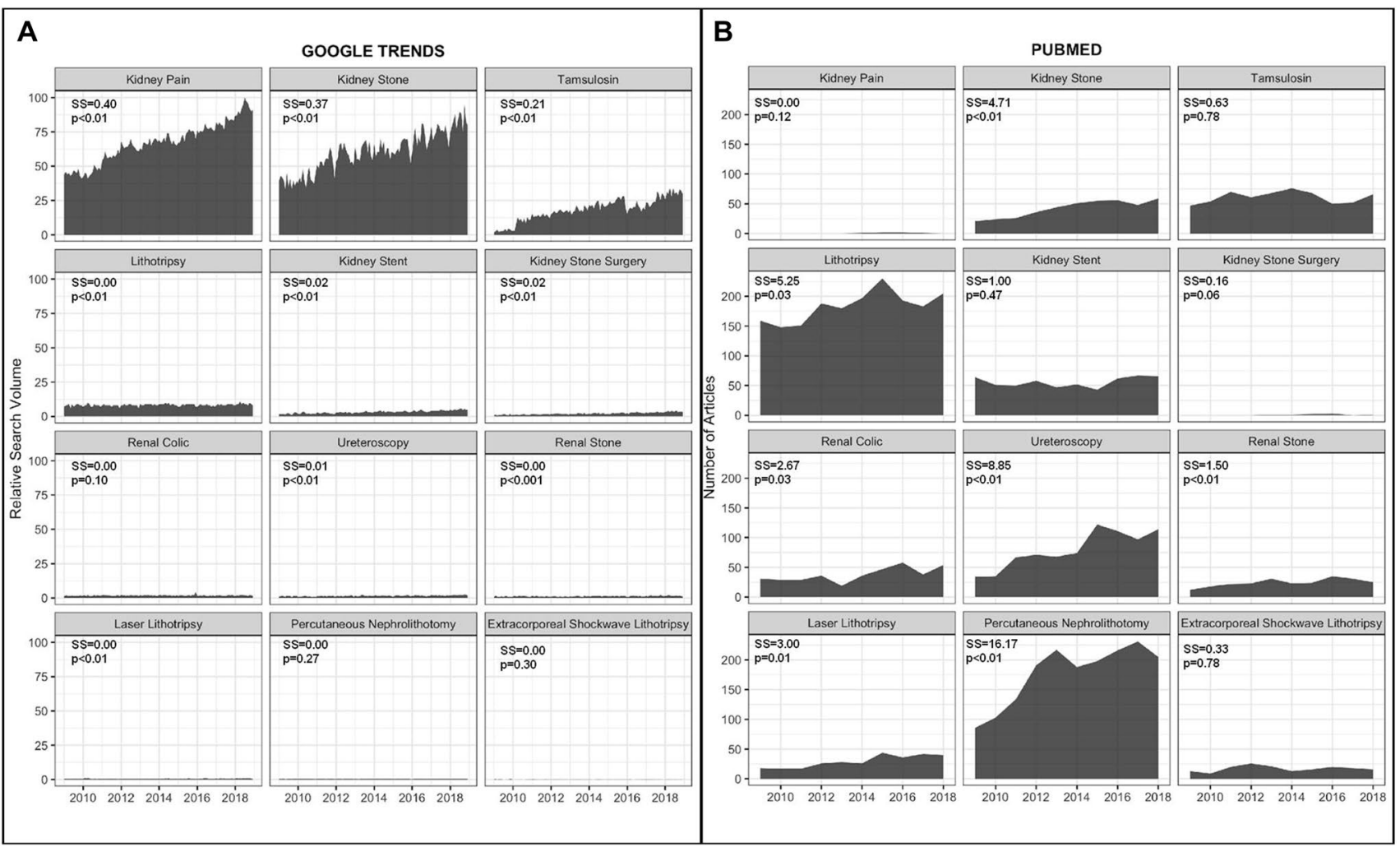

Fig. 1 Temporal Trend for each analysed stone-related term measured by Mann-Kendall Sen's Slope Estimator (SS) for Google Trend Relative Search Volume (RSV) (a) and for absolute number of publications on Pubmed (b)

surgical term, "Kidney Stone Surgery", had higher correlation as compared to the treatments above mentioned. More common and popular terms as "Kidney Stent" and "Tamsulosin" were highly correlated with "Kidney Pain" and "Kidney Stone" (Supplementary Fig. 2-the correlation is positive when there is a blue circle matching the terms in left column and upper row; when the color is more intense and the circle larger, the correlation is higher; the red circle is interpreted as no correlation between terms).

\section{Weather influence on online search patterns}

There was an eminent tendency towards higher RSV for the term "kidney stone" in the hottest states when compared to the ten coldest American states (Supplementary Fig. 3 and Supplementary Table 1). That greater public interest on the GT search for "kidney stone" achieved significance in the years 2010, 2011, 2015 and 2017. In the remainder years, a marginal difference was seen.

\section{Discussion}

This is the first study to investigate in a standardized manner with strict statistical analysis on the information-seeking patterns related to stone disease on Google Trends. In our study, we could notice a remarkable increase in interest of the patients in regard to medical information related to kidney stones in the United States.

The Google Trend platform cannot compare searching patterns of patients naive for stone treatment with those who have already been instructed in a urologic visit. Although we cannot assume the order in which an individual look for the terms online, the relative volume of search and temporal trend allow for some suppositions. Citizens probably start their research using terms that describe their symptoms or the disease itself, e.g. "kidney pain" and "kidney stone". Interestingly, "renal stone" and "renal colic" had low public interest, which demonstrate 
a cultural language preference of the name "kidney" over "renal". After looking for those terms, they possibly seek for information concerning existing clinical and surgical treatments, e.g. "tamsulosin", "lithotripsy", "kidney stent" or "kidney stone surgery". Online search volume for technical terms as "PCNL", "URS", SWL" and "laser lithotripsy" was very low. This is the first study to compare population online search behaviour with medical publication patterns. Noteworthy, our results clarify that there is a mismatch between urologic community publications on Pubmed and general information-seeking comportment. This highlights the need for a shift on media planning to better educate our patients who seek for information to make informed decision about their own health.

It is reasonable to think that there is also a significant number of patients that search for stone disease information already after their first kidney stone event or surgery. Renal colic is an emergency situation where the patient has to be rapidly assisted without the opportunity to seek for information prior to initial management. The terms "Kidney stent" was the fifth in total search volume and may reflect a post-operative information-seeking pattern. "Tamsulosin" showed expressive RSV in 2018. In total RSV, it was the third term and a significant increase was seen between 2009 and 2018 which may reflect the interest in medical management an individual might start or was already put on. Notably, tamsulosin is a medication used for other urologic disorders, e.g. benign prostatic hyperplasia, and is still considered an off-label drug for stone disease. Therefore, data must be analyzed critically.

Dreher and colleagues [8] have recently queried the Google Trends platform using terminology related to kidney stone surgical intervention in English within the United States from November 2011 through August 2017. They found "kidney stone surgery" as the most common term in comparison to "PCNL", "SWL", "URS" and "laser lithotripsy". The authors stated that research trends for the expression "kidney stone surgery" remained fairly constant over the six-year period and was more looked for in the states of Tennessee, Indiana, Ohio, Michigan and North Carolina. Likewise, they found that specific treatment modalities yielded similar search trends over time but were queried at a lower frequency than the term "kidney stone surgery". A common aspect to our study is the fact we have also found that specific surgical technical terms are indeed not relevant in regard to search volume. Nonetheless, we found that the search interest for "kidney stone surgery" increased over time and the negative results they had might be explained by the shorter period of time included in their study. If we look at our Fig. 1A, the variation of RSV for "kidney stone surgery" was not so pronounced between 2011 and 2017. Second, and more importantly, the lack of a formal statistical analysis is the main drawback of their investigation. One cannot assume just by looking at the graphic that there was no tendency in one direction or another. Finally, the authors did not investigate terms related to the disease itself and symptomatology, which we found to be the most important terminologies addressed by the population.

Forecasting epidemiology is considered an indispensable science when dealing with diseases of high prevalence or burden to the population medically and economically. Kapitány-Fövény et al. [11] investigated if measuring online activity could be used as a promising tool for forecasting infectious diseases and found a good correlation between the reported weekly case numbers of Lyme disease and search volume on GT. However, it was not completely accurate, and the authors believe that this new instrument could have a role in improving the traditional epidemiological surveillance models instead of replacing it. Verma et al. [12] studied the temporal correlation between GT and Integrated Disease Surveillance Programme data to determine if it was feasible to use online data for prediction of outbreaks or epidemics. A significant temporal association was observed, and the authors found a high correlation for Chikungunya and Dengue fever in the studied regions, while Malaria and Enteric fever had a moderate correlation, all preceding factual epidemic outbreak in 2 to 3 weeks. In that same sense, Unsal et al. [13] showed the potential utility of the GT database as a resource for epidemiologic monitoring on epistaxis and for the identification of at-risk populations. More recently, in face of the coronavirus 19 pandemic, Husnayain et al. [14] retrieved GT data for the specific locations of Taiwan. The authors found high-to-moderate correlations between GT RSV and infected cases in several regions and concluded that GT could potentially define the proper timing and site for performing risk communication strategies to the population.

The capability of GT in predicting high incidence periods of stone disease is yet to be determined. Given the high burden of the disease in regard to severity of symptoms, days lost of work, and economic expenditure, advancing with such investigation would be reasonable. Our analysis showed an increased interest in stone-related terms. By evaluating the weather influence on online search measured by GT, we could for the first time demonstrate that US states with hotter weather had a significant higher interest on the term "kidney stone" than colder states. The higher incidence of stone-related events in the US stone-belt [15] allied with our findings suggest that information-seeking behaviour follows stone disease prevalence, and this might be used for prevention and population counselling strategies.

The utility of GT use for prevention campaigns has also been investigated. Hopkins et al. [16] evaluated trends in US searches for sunscreen, sunburn, skin cancer, and melanoma and their relationships with melanoma outcomes. They found that terms "sunscreen," "sunburn," "skin cancer," 
and "melanoma" were all significantly correlated but "sunscreen" and "sunburn" had the greatest correlation. Therefore, the authors suggested that online skin cancer prevention campaigns should focus on the search terms "sunburn" and "sunscreen", given the decreasing online searches for skin cancer and melanoma, and because "sunscreen" searches were higher in areas with greater melanoma incidence. Similar prevention campaigns could be created and released prior the historical periods of increased stone-related events for any particular region of the country. Nationwide, "kidney stone" and "kidney pain" are the most indicated terms. For a particular city or state, a specific analysis might be performed to narrow key terms for that region on a determined moment.

We must acknowledge that internet use might be impacted by several factors. Of those, the grade of country development might play an important role. Interestingly, recent data show data even in underdeveloped nations, the internet access is expressive and democratic when a dictature government is not in place. As an example, according the Statista Research Department [17], of the nearly 210 million individuals in Brazil in 2016, 140 million were active internet users. Brazil was the largest internet market in Latin America and the fourth largest internet market in the world when considering the number of internet users. Also, our study included only US data where more than $90 \%$ of individuals actively access the internet. The $10 \%$ remaining are not embodied by GT numbers.

The knowledge gained with this study allows for elaboration of target content to patients seeking for information and for planning of public strategies to educate the population nationwide. Initially, prevention campaigns might focus on those keywords with greater search volumes and its effectiveness could be monitored by continuously in the same online platform. Sequentially, patient counselling and education upon specific medical terms and treatments could be performed more efficiently, allowing for a more shared-decision approach. Finally, prediction models for stone disease occurrence are a line of investigation. They could be added to the climate influence patterns confirmed by our report to increase outbreaks forecast.

\section{Conclusion}

There was a significant increase in online search for medical information related to stone disease in the US in the last decade. States with hotter weather show higher search volumes of stone-related terms than colder states. Citizens tend to look for terms related to symptoms and the disease itself. Medical management and kidney stent are expressions of particular interest to patients, while technical expressions of urologic procedures are not. Technical terms are the most expressive among stone-related scientific publications on Pubmed, highlighting the discrepancy between terminologies on both databases.

Author contributions GSM: manuscript design and conception, KVMF: manuscript design and conception, FLN: statistical analysis, FCMT: manuscript design and conception, AD: manuscript design and review, FCV: manuscript confection and review, CAB: statistical analysis, MS: manuscript design, WCN: manuscript review, EM: manuscript design and review.

Funding None.

Availability of data and material All analysed data available if necessary.

\section{Compliance with ethical standards}

Conflict of interest All authors declare that they have no conflict of interest.

Ethics approval Not necessary - online data analysis.

Consent to participate Not necessary - online data analysis.

Consent for publication Approved.

\section{References}

1. Sakhaee K (2009) Pharmacology of stone disease. Adv Chronic Kidney Dis 16:30-38

2. Moe OW (2006) Kidney stones. Pathophysiology and medical management. Lancet 367:333-344. https://doi.org/10.1016/S0140 $-6736(06) 68071-9$

3. Scales CD Jr, Smith AC, Hanley JM et al (2012) Urologic diseases in America project: prevalence of kidney stones in the United States. Eur Urol 62:160-165. https://doi.org/10.1016/j.eurur o.2012.03.052

4. Marchini GS, Mello MS, Levy R et al (2015) Contemporary trends of inpatient surgical management of stone disease: national analysis in an economic growth scenario. J Endourol 29(8):956-962. https://doi.org/10.1089/end.2015.0021

5. Geraghty RM, Jones P, Somani BK (2017) Worldwide trends of urinary stone disease treatment over the last two decades: a systematic review. J Endourol 31(6):547-556. https://doi. org/10.1089/end.2016.0895

6. Ludwig WW, Matlaga BR (2018) Urinary stone disease: diagnosis, medical therapy, and surgical management. Med Clin North Am 102(2):265-277. https://doi.org/10.1016/j.mcna.2017.10.004

7. Internet usage in United States-https://www.statista.com/topic s/2237/internet-usage-in-the-united-states/

8. Dreher PC, Tong C, Ghiraldi E et al (2018) Use of Google Trends to track online behavior and interest in kidney stone surgery. Urology 121:74-78. https://doi.org/10.1016/j.urology.2018.05.040

9. Mavragani A, Ochoa G, Tsagarakis KP (2018) Assessing the methods, tools, and statistical approaches in Google Trends research: systematic review. J Med Internet Res 20(11):270

10. What is Google Trends data-and what does it mean?-https:// medium.com/google-news-lab/what-is-google-trends-data-andwhat-does-it-mean-b48f07342ee8 
11. Kapitány-Fövény M, Ferenci T, Sulyok Z et al (2019) Can Google Trends data improve forecasting of lyme disease incidence? Zoonoses Public Health 66(1):101-107. https://doi.org/10.1111/ zph.12539

12. Verma M, Kishore K, Kumar M et al (2018) Google search Trends predicting disease outbreaks: an analysis from India. Healthc Inform Res 24(4):300-308. https://doi.org/10.4258/ hir.2018.24.4.300

13. Unsal AA, Dubal PM, Pfaff JA et al (2019) Doctor Google: correlating internet search trends for epistaxis with metropolitan climates. Am J Otolaryngol 40(3):358-363. https://doi. org/10.1016/j.amjoto.2019.02.001

14. Husnayain A, Fuad A, Su EC (2020) Applications of google search trends for risk communication in infectious disease management: a case study of COVID-19 outbreak in Taiwan. Int J Infect Dis 12(95):221-223. https://doi.org/10.1016/j.ijid.2020.03.021
15. Brikowski TH, Lotan Y, Pearle MS (2008) Climate-related increase in the prevalence of urolithiasis in the United States. Proc Natl Acad Sci USA 105:9841-9846. https://doi.org/10.1073/ pnas.0709652105

16. Hopkins ZH, Secrest AM (2019) Public health implications of Google searches for sunscreen, sunburn, skin cancer, and melanoma in the United States. Am J Health Promot 33(4):611-615. https://doi.org/10.1177/0890117118811754

17. Statista Research Department: https://www.statista.com/

Publisher's Note Springer Nature remains neutral with regard to jurisdictional claims in published maps and institutional affiliations. 